PHYSICAL REVIEW D 76, 035005 (2007)

\title{
String-derived $D_{4}$ flavor symmetry and phenomenological implications
}

\author{
Pyungwon Ko \\ School of Physics, KIAS, Cheongnyangni-dong, Seoul, 130-722, Korea \\ Tatsuo Kobayashi \\ Department of Physics, Kyoto University, Kyoto 606-8502, Japan \\ Jae-hyeon Park \\ Department of Physics, Tohoku University, Sendai 980-8578, Japan \\ Stuart Raby \\ Department of Physics, The Ohio State University, Columbus, Ohio 43210 USA \\ (Received 1 May 2007; published 17 August 2007; corrected 24 August 2007)
}

\begin{abstract}
In this paper we show how some flavor symmetries may be derived from the heterotic string, when compactified on a $6 \mathrm{D}$ orbifold. In the body of the paper we focus on the $D_{4}$ family symmetry, recently obtained in $Z_{3} \times Z_{2}$ orbifold constructions. We show how this flavor symmetry constrains fermion masses, as well as the soft SUSY breaking mass terms. Flavor symmetry breaking can generate the hierarchy of fermion masses and at the same time the flavor symmetry suppresses large flavor changing neutral current processes.
\end{abstract}

DOI: 10.1103/PhysRevD.76.035005

PACS numbers: 11.25.Wx, 12.15.Ff, 12.60.Jv

\section{INTRODUCTION}

Fermion masses and mixing angles are the precision low energy data which will test any new physics beyond the standard model. Quarks and leptons come in three flavors (or families) with a distinct hierarchy of masses for charged fermions; with the third family heavier than the second, which is heavier than the first. Moreover the mixing angles evident in charge current electroweak processes are small and favor nearest neighbor mixing with respect to family number. In the neutrino sector, the situation is not as clear. There are possibly more than three light neutrinos. Their masses may be Majorana or Dirac. Even if one assumes just three light Majorana neutrinos, a normal or inverted hierarchy is possible. Finally the leptonic mixing angles, so-called Pontecorvo-Maki-Nakagawa-Sakita (PMNS) angles, (analogs of the Cabibbo-KobayashiMaskawa (CKM) mixing for quarks) are large, with maximal mixing $\left(\sim 45^{\circ}\right)$ between $\nu_{\mu}-\nu_{\tau}$ and large mixing ( $\sim$ $\left.30^{\circ}\right)$ between $\nu_{e}-\left(\nu_{\mu}, \nu_{\tau}\right)$. Within the context of the seesaw mechanism, this may be generated via large mixing in the Dirac neutrino mass matrix or in the right-handed Majorana mass matrix [1]. However Ref. [2] showed that bilarge neutrino mixing may also be obtained with completely hierarchical Dirac and Majorana neutrino mass matrices. In a recent paper this analysis was extended to supersymmetric $S O(10)$ [3].

Understanding the origin of fermion flavor structure, i.e. fermion masses and mixing angles, is one of the important issues in particle physics. In field-theoretical model building, one often assumes certain types of flavor symmetries, which control Yukawa couplings and higher dimensional operators. Higher dimensional operators are useful as effective Yukawa couplings when proper scalar fields de- velop their expectation values (VEVs). Continuous and discrete non-Abelian symmetries, e.g. $U(2), D_{4}, S_{3}(\approx$ $\left.D_{3}\right), A_{4}, Q_{n}, \Delta\left(3 n^{2}\right), \Delta\left(6 n^{2}\right)$ are assumed as flavor symmetries [3-11], while Abelian symmetries such as $U(1)$, $Z_{N}$ are often assumed, too. However, from the viewpoint of 4D field theory their origins are not clear.

Such flavor structure also has significant implications for supersymmetry (SUSY) breaking terms [12]. Superpartners have not been detected yet, but flavor changing neutral current (FCNC) processes are strongly constrained by experiments $[13,14]^{1}$; requiring that soft SUSY breaking terms should be approximately degenerate between the first and second families. Thus, in several field-theoretical models the first and second families are assumed to be a doublet under certain flavor symmetries, while the third family may be a singlet. Furthermore, there are FCNC constraints for the third family, although not as restrictive as for the first and second families.

Superstring theory is a promising candidate for a unified theory including gravity. Hence, it is very important to study what type of flavor structures can be realized within the framework of string models and to investigate their implications for particle physics. Heterotic orbifold models can lead to realistic 4D models, and one interesting feature is that phenomenological aspects are determined by geometrical properties of the orbifolds. For examples, in Refs. [16-19] $Z_{3}$ models with three families have been obtained. In $Z_{3}$ orbifold models, the untwisted matter sector has the degeneracy factor three, which originates from a triplet of $S U(3)_{H}$ holonomy; a subgroup of broken $S U(4)_{R}$ symmetry. Also each $2 \mathrm{D} Z_{3}$ orbifold of a $6 \mathrm{D}$

\footnotetext{
${ }^{1}$ See also [15] and references therein.
} 
orbifold has three fixed points, with a degenerate massless spectra, unless one introduces Wilson lines. These triplets in the untwisted and twisted sectors correspond to three families in the models of Refs. [16-19]. In this case, the Higgs fields must also be a triplet such that at least the top Yukawa coupling is allowed as a 3-point coupling.

Recently, a new type of model has been constructed on the $Z_{2} \times Z_{3}=Z_{6}$ orbifold $[20,21] .^{2}$ In these models, three families are realized as a singlet and doublet under a $D_{4}$ flavor symmetry. That is these models are, as far as we know, the first models to realize such flavor structure. The third family of quarks and leptons are $D_{4}$ singlets. Thus, one important aspect of such flavor structure is that the Higgs field, which is allowed to couple to the top quark, is a singlet under the flavor symmetry. Moreover, the flavor structure has other significant implications in the Yukawa matrices and SUSY breaking terms. Hence, it is important to study phenomenological aspects of the string-derived $D_{4}$ flavor symmetry as well as other discrete non-Abelian flavor symmetries.

Furthermore, in Ref. [23], all possible non-Abelian discrete flavor symmetries, which can appear from heterotic orbifold models, have been classified. Those include $D_{4}$, $\Delta(54)$ and $S W_{4}$, and $\Delta(54)$ can break into $D_{3}$. In addition, it has been shown that the model on $S^{1} / Z_{2}$ can include only $D_{4}$-doublets and trivial singlets as fundamental modes, but models on $T^{2} / Z_{4}$ and $T^{4} / Z_{8}$ can include nontrivial $D_{4}$ singlets, too.

Here we study the $D_{4}$ flavor structure. Generic heterotic orbifold models leading to the $D_{4}$ flavor structure are considered. We also analyze their phenomenological aspects, that is, effective Yukawa couplings and SUSY breaking terms. Furthermore we study FCNC constraints on SUSY breaking terms in detail.

This paper is organized as follows. In Sec. II, we show how the $D_{4}$ flavor structure appears from heterotic orbifold models. In Sec. III, we study its implication on Yukawa matrices. In Sec. IV, we study predictions of SUSY breaking squark masses and scalar trilinear couplings. Section V is devoted to conclusion and discussion. In Appendix A, group-theoretical aspects of $D_{4}$ are summarized. In Appendix B, we summarize sfermion masses and scalar trilinear couplings, derived from a $U(1)$ Froggatt-Nielsen model to compare with our model. In Appendix C, we comment on a possibility to realize SUSY breaking terms consistent with $t-b-\tau$ Yukawa unification.

\section{II. $D_{4}$ FLAVOR STRUCTURE IN STRING MODELS}

\section{A. $D_{\mathbf{4}}$ flavor symmetry from orbifold models}

In this section, we show that the $D_{4}$ flavor structure can be derived in $Z_{2} \times Z_{M}$ heterotic orbifold models. (See also [23].) The $Z_{2} \times Z_{M}$ orbifold is obtained as follows. First,

\footnotetext{
${ }^{2}$ See also for recent studies on model construction [22].
}

we consider the 6D $T^{2} \times T^{2} \times T^{2}$ torus. Then, we divide it by two independent twists, $\theta$ and $\omega$, whose eigenvalues are $e^{2 \pi i v_{1}}$ and $e^{2 \pi i v_{2}}$ with

$$
v_{1}=(1 / 2,-1 / 2,0), \quad v_{2}=(0,1 / M,-1 / M),
$$

respectively, in the complex basis, such that we obtain the $Z_{2} \times Z_{M}$ orbifold preserving $N=14 \mathrm{D}$ SUSY. Note that the first plane becomes the $2 \mathrm{D} Z_{2}$ orbifold by dividing the $T^{2}$ by the $Z_{2}$ twist. The $2 \mathrm{D} Z_{2}$ orbifold has four fixed points denoted as $\left(\frac{n_{1}}{2} \mathbf{e}_{1}+\frac{n_{2}}{2} \mathbf{e}_{2}\right)$ for $n_{1}, n_{2}=0,1$, where $\mathbf{e}_{1}$ and $\mathbf{e}_{2}$ are lattice vectors defining $T^{2}$. The twisted states are associated with these fixed points. Namely, the $\theta^{2 k+1} \omega^{\ell}$ twisted states have the degenerate spectrum for these four fixed points, while for the $\theta^{2 k} \omega^{\ell}$ twist this space is just the fixed torus and the degeneracy factor from this space is just one.

We can introduce degree-two Wilson lines $2 W_{i}=\Gamma_{G}$ associated with $\mathbf{e}_{i}(i=1,2)$, where $\Gamma_{G}$ is the gauge lattice, i.e. the $E_{8} \times E_{8}$ lattice for the $E_{8} \times E_{8}$ heterotic string theory. For example, the nonvanishing Wilson line $W_{1}$ resolves the degeneracy between the fixed points $\frac{n_{2}}{2} \mathbf{e}_{2}$ and $\frac{1}{2} \mathbf{e}_{1}+\frac{n_{2}}{2} \mathbf{e}_{2}\left(n_{2}=0,1\right)$, that is, the massless spectrum of the twisted states corresponding to the fixed points $\frac{n_{2}}{2} \mathbf{e}_{2}$ differs from one corresponding to the fixed points $\frac{1}{2} \mathbf{e}_{1}+$ $\frac{n_{2}}{2} \mathbf{e}_{2}\left(n_{2}=0,1\right)$. However, there still remains the degeneracy factor two unless we introduce a nontrivial Wilson line $W_{2}$ along the $\mathbf{e}_{2}$ direction. Thus, the degeneracy between the states $\left|n_{2}=0\right\rangle$ and $\left|n_{2}=1\right\rangle$ in the $\theta^{2 k+1} \omega^{\ell}$ twisted sector is the origin of doublets under our flavor symmetry, studied in this paper. We will show later that the flavor symmetry is actually the $D_{4}$ symmetry. On the other hand, the $\theta^{2 k} \omega^{\ell}$ twisted sector as well as the untwisted sector does not have such a fixed point structure. Hence, the states in such sectors correspond to a singlet under our flavor symmetry.

Here we show that the above flavor structure corresponds to the $D_{4}$ flavor symmetry. The Lagrangian has the permutation symmetry between the states $\left|n_{2}\right\rangle$ with $n_{2}=0,1$. In addition, each coupling is controlled by the $Z_{2}$ symmetry, under which the state $\left|n_{2}\right\rangle$ is transformed as $\left|n_{2}\right\rangle \rightarrow(-1)^{n_{2}}\left|n_{2}\right\rangle$. These transformations are denoted by the two Pauli matrices,

$$
\sigma_{1}=\left(\begin{array}{cc}
0 & 1 \\
1 & 0
\end{array}\right), \quad \sigma_{3}=\left(\begin{array}{cc}
1 & 0 \\
0 & -1
\end{array}\right)
$$

respectively, on the state basis $\left(\left|n_{2}=0\right\rangle,\left|n_{2}=1\right\rangle\right)$. The complete closed set of operations forms the discrete nonAbelian $D_{4}$ symmetry, which consists of

$$
\pm I, \quad \pm \sigma_{1}, \quad \pm i \sigma_{2}, \quad \pm \sigma_{3} .
$$

The $D_{4}$ symmetry is a symmetry of a square. Thus, the $\theta^{2 k+1} \omega$ twisted states are $D_{4}$ doublets, while the other $\theta^{2 k} \omega$ twisted states and the untwisted states are $D_{4}$ singlets. 
Note, a field-theoretical orbifold grand unified theory (GUT) explanation may be useful for field theory model builders. We consider the model with the extra dimension $S^{1} / Z_{2}$, which has two fixed points. String theory requires that brane fields on these two fixed points must be degenerate in the massless spectrum unless a nonvanishing Wilson line is introduced to resolve this degeneracy. Brane fields on two fixed points of $S^{1} / Z_{2}$ are $D_{4}$ doublets corresponding to $\theta^{2 k+1} \omega$ twisted states in $Z_{2} \times Z_{M}$ heterotic orbifold models. On the other hand, bulk fields on $S^{1} / Z_{2}$ are $D_{4}$ singlets corresponding to $\theta^{2 k} \omega$ twisted states and untwisted states. Finally string selection rules require that the superpotential contains an even number of doublet fields at each fixed point.

\section{B. Explicit model}

Here we give the example with the $D_{4}$ flavor structure, which has been obtained in Ref. [21]. That is the $Z_{2} \times$ $Z_{3}=Z_{6}$ orbifold model with the Pati-Salam gauge group $S U(4) \times S U(2)_{L} \times S U(2)_{R}$ and the extra gauge group $S O(10)^{\prime} \times S U(2)^{\prime} \times U(1)^{5}$. The model has three families under the Pati-Salam group, i.e. $3 \times[(\mathbf{4}, \mathbf{2}, \mathbf{1})+(\overline{\mathbf{4}}, \mathbf{1}, \mathbf{2})]$. The untwisted sector has one family, $(\mathbf{4}, \mathbf{2}, \mathbf{1})+(\overline{\mathbf{4}}, \mathbf{1}, \mathbf{2})$ under $S U(4) \times S U(2)_{L} \times S U(2)_{R}$, which include the third family of left-handed quarks and antiquarks, $q_{3}, \bar{u}_{3}$, and $\bar{d}_{3}$. The $\theta \omega$ twisted sector has the other two families, which include the first and second families of quarks, $q_{i}, \bar{u}_{i}$ and $\bar{d}_{i}$ $(i=1,2)$. The Higgs field $(\mathbf{1}, \mathbf{2}, \mathbf{2})$ comes from the untwisted sector, and it includes the up-sector and downsector Higgs fields, $h_{u}$ and $h_{d}$. Thus, the third family of quarks as well as Higgs fields are $D_{4}$ singlets, while the other two families are $D_{4}$ doublets. In Table I, we show their extra $U(1)$ charges for later convenience. This model also includes extra matter fields usually found in string models. (See for details Ref. [21].)

The discussion in the rest of the paper is quite generic and independent of the particular gauge group, since we are now more interested in the consequences of the flavor symmetries. Therefore, we will discuss the case that three families of quarks and leptons in the basis of the standard model gauge group consist of singlets and doublets under the $D_{4}$ flavor symmetry. In the following sections, we concentrate on the phenomenological implications of the $D_{4}$ flavor structure in the quark sector.

TABLE I. Extra $U(1)$ charges in explicit string model.

\begin{tabular}{lrrrrr}
\hline \hline & $Q_{1}$ & $Q_{2}$ & $Q_{3}$ & $Q_{4}$ & $Q_{A}$ \\
\hline$q_{3}$ & 1 & 1 & 0 & 3 & -2 \\
$q_{1,2}$ & -1 & 0 & 0 & 0 & 0 \\
$\bar{u}_{3}, \bar{d}_{3}$ & -3 & 0 & 0 & -1 & 0 \\
$\bar{u}_{1,2}, \bar{d}_{1,2}$ & -1 & 0 & 0 & 0 & 0 \\
$h_{u}, h_{d}$ & 2 & -1 & 0 & -2 & 2 \\
\hline \hline
\end{tabular}

\section{YUKAWA MATRICES}

In this section and the next section, we study phenomenological implications of the $D_{4}$ flavor structure. First, in this section we consider Yukawa matrices. We consider the $D_{4}$ flavor structure where the third family $q_{3}, \bar{u}_{3}, \bar{d}_{3}$ corresponds to the $D_{4}$ trivial singlet $A_{1}$ and the first and second families $q_{i}, \bar{u}_{i}, \bar{d}_{i}(i=1,2)$ are $D_{4}$ doublets. The up- and down-sectors of Higgs fields are also $D_{4}$ singlets. (See Appendix A for more details on the discrete group $D_{4}$ and its representations.)

Let us examine the 3-point couplings,

$$
y_{i j}^{(u)} q_{i} \bar{u}_{j} H_{u}, \quad y_{i j}^{(d)} q_{i} \bar{d}_{j} H_{d}
$$

The $D_{4}$ algebra allows diagonal entries, i.e. $y_{i j}^{(u, d)}=$ $y_{i}^{(u, d)} \delta_{i j}$ with $y_{1}^{(u, d)}=y_{2}^{(u, d)} \neq y_{3}^{(u, d)}$. If $y_{i}^{(u, d)}=O(1)$, that is not realistic. Thus, we assume that extra symmetries allow the couplings of the third family, but not the first or second families, i.e.

$$
y^{(u, d)}=\left(\begin{array}{ccc}
0 & 0 & 0 \\
0 & 0 & 0 \\
0 & 0 & y_{33}^{(u, d)}
\end{array}\right) .
$$

Actually, the example shown in Sec. II B has extra $U(1)$ symmetries which forbid the Yukawa couplings for the first and second families, but allow the third family Yukawa couplings. In the explicit string model, other stringy selection rules also forbid the Yukawa couplings for the first and second families [21]. Furthermore, in the model [21], the third family Yukawa couplings are required to be the same as the gauge coupling $g$, i.e. $y_{33}^{(u, d)}=g \approx O(1)$.

Now, let us consider how to generate the other entries of the Yukawa matrix. Those are expected to be generated as effective Yukawa couplings through higher dimensional operators once certain scalar fields develop their VEVs. String models, in general, have several gauge-singlet fields $\sigma$, and they can develop VEVs along flat directions. Of course, each gauge-singlet field transforms as a trivial singlet $A_{1}$ or a doublet under the $D_{4}$ group. In addition, a product of $D_{4}$ doublets include four types of $D_{4}$-singlets, $A_{1}, B_{1}, B_{2}$, and $A_{2}$. Thus, higher dimensional operators can generate the following effective Yukawa matrices,

$$
y^{(u, d)}=\left(\begin{array}{ccc}
\sigma_{\left[A_{1}\right]}^{(u, d)}+\sigma_{\left[B_{2}\right]}^{(u, d)} & \sigma_{\left[B_{1}\right]}^{(u, d)}+\sigma_{\left[A_{2}\right]}^{(u, d)} & \sigma_{\left[D_{1}\right]}^{(u, d)} \\
\sigma_{\left[B_{1}\right]}^{(u, d)}-\sigma_{\left[A_{2}\right]}^{(u, d)} & \sigma_{\left[A_{1}\right]}^{(u, d)}-\sigma_{\left[B_{2}\right]}^{(u, d)} & \sigma_{\left[D_{2}\right]}^{(u, d)} \\
\sigma_{\left[D_{1}^{\prime}\right]}^{(u, d)} & \sigma_{\left[D_{2}^{\prime}\right]}^{(u, d)} & 1
\end{array}\right),
$$

up to $O(1)$ coefficients (assuming the singlet fields have proper $U(1)$ charges). Here, $\sigma_{[R]}^{(u, d)}$ denotes a product of gauge singlets in the $R$ representation under $D_{4}$, and $\sigma_{\left[D_{1}\right]}^{(u, d)}$ and $\sigma_{\left[D_{2}\right]}^{(u, d)}$ are $D_{4}$ doublet. ${ }^{3}$ Here, their VEVs are denoted

\footnotetext{
${ }^{3}$ In the explicit model [21] we can generate such effective Yukawa matrices by SM gauge singlets, contained in the model.
} 
by dimensionless parameters with units $M=1$, where $M$ is the Planck scale. These effective Yukawa matrices have more than enough free parameters (as VEVs of singlets $\sigma$ ), such that one can realize realistic values of quark masses and mixing angles in a generic model. In other words, we have no prediction in the Yukawa sector using only the $D_{4}$ flavor symmetry, unless additional symmetries or conditions for $\sigma$ are imposed. Note, for later reference, the experimental values of the quark mass ratios and mixing angles can be given by the approximate relations

$$
\begin{aligned}
& \frac{m_{d}}{m_{s}} \sim \lambda^{2}, \quad \frac{m_{s}}{m_{b}} \sim \frac{1}{2} \lambda^{2}, \\
& \frac{m_{u}}{m_{c}} \sim \lambda^{4}, \quad \frac{m_{c}}{m_{t}} \sim \lambda^{3}-\lambda^{4}, \\
& V_{u s} \sim \lambda, \quad V_{c b} \sim \lambda^{2}, \quad V_{u b} \sim \lambda^{3}-\lambda^{4},
\end{aligned}
$$

where $\lambda=0.22$.

\section{Model}

Here we show a simple example leading to realistic results. We introduce $\sigma_{\left[D_{1,2}\right]}^{(q)}, \sigma_{\left[D_{1,2}\right]}^{(d)}, \hat{\sigma}_{\left[D_{1,2}\right]}^{(d)}$, and $\sigma_{[A 1]}$ (unrelated to the variables $\sigma^{(u, d)}$ introduced in Eq. (3.3)), and assume they develop VEVs. $\sigma_{[A 1]}$ is a $D_{4}$ trivial singlet and
TABLE II. $U(1)$ charges of the fields.

\begin{tabular}{lcc}
\hline \hline & $Q_{1}$ & $Q_{2}$ \\
\hline$q_{3}$ & 1 & 3 \\
$q_{1,2}$ & -1 & 0 \\
$\bar{u}_{3}$ & -3 & -1 \\
$\bar{u}_{1,2}$ & -1 & 0 \\
$\bar{d}_{3}$ & $-3-a$ & $-1-b$ \\
$\bar{d}_{1,2}$ & -1 & 0 \\
$h_{u}$ & 2 & -2 \\
$h_{d}$ & 2 & -2 \\
$\sigma_{\left[D_{1,2}\right]}^{(q)}$ & 2 & 3 \\
$\sigma_{\left[D_{1,2}\right]}^{(d)}$ & $2+x$ & $3+y$ \\
$\hat{\sigma}_{\left[D_{1,2}\right]}^{(d)}$ & $-2-x$ & $-1-y$ \\
$\sigma_{[A 1]}$ & $a$ & $b$ \\
\hline \hline
\end{tabular}

the others are $D_{4}$ doublets. We also introduce two extra $U(1)$ symmetries. Note, the $U(1)$ charges of each field are assigned in Table II. The following discussion is independent of values of the $U(1)$ symmetry parameters $a, b, x$, and $y$ (see Table II). A working example is

$$
a=1, \quad b=0, \quad x=0, \quad y=1 .
$$

Then we obtain the following forms of Yukawa matrices,

$$
\begin{aligned}
& y^{(u)}=\left(\begin{array}{ccc}
c_{a}^{(u)} \sigma_{\left[D_{1}\right]}^{(d)} \hat{\sigma}_{\left[D_{1}\right]}^{(d)}+c_{b}^{(u)} \sigma_{\left[D_{2}\right]}^{(d)} \hat{\sigma}_{\left[D_{2}\right]}^{(d)} & c_{b}^{(u)}\left(\sigma_{\left[D_{1}\right]}^{(d)} \hat{\sigma}_{\left[D_{2}\right]}^{(d)}+\sigma_{\left[D_{2}\right]}^{(d)} \hat{\sigma}_{\left[D_{1}\right]}^{(d)}\right) & c_{3}^{(u)} \sigma_{\left[D_{1}\right]}^{(q)} \\
c_{b}^{(u)}\left(\sigma_{\left[D_{1}\right]}^{(d)} \hat{\sigma}_{\left[D_{2}\right]}^{(d)}+\sigma_{\left[D_{2}\right]}^{(d)} \hat{\sigma}_{\left[D_{1}\right]}^{(d)}\right) & c_{b}^{(u)} \sigma_{\left[D_{1}\right]}^{(d)} \hat{\sigma}_{\left[D_{1}\right]}^{(d)}+c_{a}^{(u)} \sigma_{\left[D_{2}\right]}^{(d)} \hat{\sigma}_{\left[D_{2}\right]}^{(d)} & c_{3}^{(u)} \sigma_{\left[D_{2}\right]}^{(q)} \\
0 & \sigma_{1}^{(q)}
\end{array}\right), \\
& y^{(d)}=\left(\begin{array}{ccc}
c_{a}^{(d)} \sigma_{\left[D_{1}\right]}^{(d)} \hat{\sigma}_{\left[D_{1}\right]}^{(d)}+c_{b}^{(d)} \sigma_{\left[D_{2}\right]}^{(d)} \hat{\sigma}_{\left[D_{2}\right]}^{(d)} & c_{b}^{(d)}\left(\sigma_{\left[D_{1}\right]}^{(d)} \hat{\sigma}_{\left[D_{2}\right]}^{(d)}+\sigma_{\left[D_{2}\right]}^{(d)} \hat{\sigma}_{\left[D_{1}\right]}^{(d)}\right) & c_{3}^{(d)} \sigma_{\left[D_{1}\right]}^{(q)} \sigma_{[A 1]} \\
c_{b}^{(d)}\left(\sigma_{\left[D_{1}\right]}^{(d)} \hat{\sigma}_{\left[D_{2}\right]}^{(d)}+\sigma_{\left[D_{2}\right]}^{(d)} \hat{\sigma}_{\left[D_{1}\right]}^{(d)}\right) & c_{b}^{(d)} \sigma_{\left[D_{1}\right]}^{(d)} \hat{\sigma}_{\left[D_{1}\right]}^{(d)}+c_{a}^{(d)} \sigma_{\left[D_{2}\right]}^{(d)} \hat{\sigma}_{\left[D_{2}\right]}^{(d)} & c_{3}^{(d)} \sigma_{\left[D_{2}\right]}^{(q)} \sigma_{[A 1]} \\
0 & \sigma_{[A 1]}
\end{array}\right) .
\end{aligned}
$$

Here the $(3,1)$ and $(3,2)$ entries in both Yukawa matrices of up and down sectors are quite suppressed, and irrelevant to the following discussions.

We choose the VEVs of the $\sigma$ fields as

$$
\begin{gathered}
\sigma_{\left[D_{1}\right]}^{(q)} \sim \lambda^{3}, \quad \sigma_{\left[D_{2}\right]}^{(q)} \sim \lambda^{2}, \\
\sigma_{\left[D_{1}\right]}^{(d)} \sim \sigma_{\left[D_{2}\right]}^{(d)} \sim \hat{\sigma}_{\left[D_{1}\right]}^{(d)} \sim \hat{\sigma}_{\left[D_{2}\right]}^{(d)} \sim \lambda^{2}, \quad \sigma_{[A 1]} \sim \lambda .
\end{gathered}
$$

Then, we have naturally the following texture,

$$
y^{(u)} \sim\left(\begin{array}{ccc}
\lambda^{4} & \lambda^{4} & \lambda^{3} \\
\lambda^{4} & \lambda^{4} & \lambda^{2} \\
0 & 0 & 1
\end{array}\right), \quad y^{(d)} \sim\left(\begin{array}{ccc}
\lambda^{4} & \lambda^{4} & \lambda^{4} \\
\lambda^{4} & \lambda^{4} & \lambda^{3} \\
0 & 0 & \lambda
\end{array}\right) .
$$

This texture can fit the quark mass ratios, the CKM mixing angles, and the KM phase. Note, however, the mass ratios, $m_{u} / m_{c}$ and $m_{d} / m_{s}$, and the mixing angle $V_{u s}$, are expected naturally to satisfy $m_{u} / m_{c} \approx m_{d} / m_{s} \approx V_{u s}=O(1)$ unless we fine-tune coefficients. The upper left $2 \times 2$ submatrices are in democratic forms. To realize the mass hierarchy between the first and the second family quarks, we need the following fine-tuning of the upper left $2 \times 2$ submatrices for the up and down sectors, $y_{(22)}^{(u)}$ and $y_{(22)}^{(d)}$,

$$
\begin{aligned}
& y_{(22)}^{(u)}=O\left(\lambda^{4}\right)\left(\begin{array}{ll}
1+\varepsilon_{11}^{(u)} & 1+\varepsilon_{12}^{(u)} \\
1+\varepsilon_{12}^{(u)} & 1+\varepsilon_{22}^{(u)}
\end{array}\right), \\
& y_{(22)}^{(d)}=O\left(\lambda^{4}\right)\left(\begin{array}{ll}
1+\varepsilon_{11}^{(d)} & 1+\varepsilon_{12}^{(d)} \\
1+\varepsilon_{12}^{(d)} & 1+\varepsilon_{22}^{(d)}
\end{array}\right),
\end{aligned}
$$

with

$$
\begin{aligned}
& \varepsilon_{11}^{(u)}-2 \varepsilon_{12}^{(u)}+\varepsilon_{22}^{(u)}=O\left(\lambda^{4}\right), \\
& \varepsilon_{11}^{(d)}-2 \varepsilon_{12}^{(d)}+\varepsilon_{22}^{(d)}=O\left(\lambda^{2}\right) .
\end{aligned}
$$


At any rate, we have a sufficient number of parameters to realize the above fine-tuning.

\section{SUSY BREAKING TERMS}

Here we study SUSY breaking terms in the model with the $D_{4}$ flavor structure. In particular, we are interested in the forms of sfermion mass-squared matrices and $A$-terms, and study their degeneracies.

\section{A. Sfermion masses}

Let us first study squark masses. It is obvious that before the $D_{4}$ flavor symmetry breaks the $D_{4}$ flavor structure leads to the soft scalar mass-squared matrices,

$$
m_{\phi_{i} \phi_{j}}^{2}=\left(\begin{array}{ccc}
m_{\phi_{1} \phi_{1}}^{2} & 0 & 0 \\
0 & m_{\phi_{1} \phi_{1}}^{2} & 0 \\
0 & 0 & m_{\phi_{3} \phi_{3}}^{2}
\end{array}\right),
$$

for $\phi_{i}=q_{i}, \bar{u}_{i}, \bar{d}_{i}(i=1,2,3)$. However, we are interested in corrections to the above form from $D_{4}$ flavor symmetry breaking, and we estimate such corrections in what follows.

We consider the SUSY breaking scenario, where moduli fields $M$ including the dilaton are dominant in SUSY breaking [24-26]. Such a scenario would be plausible within the framework of string-inspired supergravity.

Before flavor symmetry breaking, the $D_{4}$ flavor symmetry requires that the Kähler potential of matter fields has the diagonal form

$$
K_{\text {matter }}=\sum_{\phi_{i}=q_{i}, u_{i}, d_{i}} K_{\phi_{i} \phi_{i}^{\dagger}}(M)\left|\phi^{i}\right|^{2},
$$

with

$$
K_{\phi_{1} \phi_{1}^{\dagger}}(M)=K_{\phi_{2} \phi_{2}^{\dagger}}(M),
$$

where $\phi_{i}=q_{i}, u_{i}, d_{i}$. In general, the Kähler metric $K_{\phi_{i} \phi_{i}^{\dagger}}$ depends on moduli fields $M$.

In general, we obtain the following soft SUSY breaking scalar masses [27],

$$
m_{\phi_{i} \phi_{i}}^{2}=V_{0}+m_{3 / 2}^{2}-\sum_{a, b} F^{\Phi_{a}} \bar{F}^{\Phi_{b}} \partial_{\Phi_{a}} \partial_{\bar{\Phi}_{b}} \ln \left(K_{\phi_{i} \phi_{i}^{\dagger}}\right),
$$

where $V_{0}$ is the vacuum energy and $m_{3 / 2}$ is the gravitino mass defined by the total Kähler potential $K$ and superpotential $W$ as $m_{3 / 2}^{2} \equiv\left\langle e^{K}|W|^{2}\right\rangle$. Thus, the $D_{4}$ flavor structure leads to the soft scalar mass-squared matrices (4.1) for $\phi_{i}=q_{i}, \bar{u}_{i}, \bar{d}_{i}(i=1,2,3)$. Naturally, nonvanishing entries, $m_{\phi_{i} \phi_{i}}^{2}$ are of $O\left(m_{3 / 2}^{2}\right)$.

The $D_{4}$ breaking induces off-diagonal entries of the Kähler metric and squark mass-squared matrices. The (1, 2) entry of the Kähler metric for $\phi_{i}=q_{i}, \bar{u}_{i}, \bar{d}_{i}$ can be induced by e.g.

$$
C_{\phi_{1} \phi_{2}^{\dagger}}(M) \sigma_{[D 1]}^{(d)} \sigma_{[D 2]}^{(d) \dagger} \phi_{1} \phi_{2}^{\dagger} \text {, }
$$

and other similar operators, where the coefficient $C_{\phi_{1} \phi_{2}^{\dagger}}(M)$ may depend on moduli $M$. Similarly, the $(2,1)$ entry can be induced. Furthermore, the $(i, 3)$ and $(3, i)$ entries $(i=1,2)$ for left-handed squarks can be induced by

$$
C_{\phi_{i} \phi_{3}^{\dagger}}(M) \sigma_{[D i]}^{(q)} q_{i} q_{3}^{\dagger}, \quad C_{\phi_{3} \phi_{i}^{\dagger}}(M) \sigma_{[D i]}^{(q) \dagger} q_{3} q_{i}^{\dagger} .
$$

These corrections are dominant. Although other terms are allowed, those are not important in the following discussion.

This Kähler metric generates the following form of lefthanded squark masses squared in the flavor basis,

$$
m_{q}^{2}=\left(\begin{array}{ccc}
m_{q_{1} q_{1}}^{2} & O\left(\lambda^{4} m^{2}\right) & O\left(\lambda^{3} m^{2}\right) \\
O\left(\lambda^{4} m^{2}\right) & m_{q_{1} q_{1}}^{2} & O\left(\lambda^{2} m^{2}\right) \\
O\left(\lambda^{3} m^{2}\right) & O\left(\lambda^{2} m^{2}\right) & m_{q_{3} q_{3}}^{2}
\end{array}\right),
$$

where $m$ would be of the same order as $m_{q_{1} q_{1}}$ and $m_{q_{3} q_{3}}$. Similarly, down-sector right-handed squark masses are obtained as

$$
m_{d}^{2}=\left(\begin{array}{ccc}
m_{d_{1} d_{1}}^{2} & O\left(\lambda^{4} m^{2}\right) & 0 \\
O\left(\lambda^{4} m^{2}\right) & m_{d_{1} d_{1}}^{2} & 0 \\
0 & 0 & m_{d_{3} d_{3}}^{2}
\end{array}\right),
$$

where $(i, 3)$ and $(3, i)$ entries are suppressed sufficiently. The up-sector right-handed squark masses have the same form.

Note that $F$-components $F_{\sigma}$ of $\sigma$ fields as well as moduli $F$-terms contribute to squark masses. Both contributions lead to the above form of squark masses squared, because we have

$$
F_{\sigma[R]}^{\dagger}=-e^{\langle K\rangle / 2}\left\langle K_{\sigma[R] \sigma[R]} \sigma_{[R]}^{\dagger} \hat{W}+\partial_{\sigma[R]} \hat{W}\right\rangle,
$$

where $K_{\sigma[R] \sigma[R]}$ denotes the Kähler metric of $\sigma_{[R]}$, and $\hat{W}$ is the nontrivial superpotential leading to SUSY breaking, and naturally we estimate $F_{\sigma} / \sigma=O\left(m_{3 / 2}\right)$. $F$-components of $\sigma$-fields are more important for estimating $A$-terms, and we will discuss them in more detail in the next subsection.

Here we define mass insertion parameters $\left(\delta_{i j}^{u, d}\right)_{X Y}$, i.e.,

$$
\left(\delta_{i j}^{u, d}\right)_{X Y} \equiv \frac{\left(m_{i j}^{u, d}\right)_{X Y}^{2}}{\tilde{m}^{2}}
$$

where $X Y=L L, R R, L R$ and $\tilde{m}^{2}$ denotes the average squark mass-squared. 
Our model leads to

$$
\begin{aligned}
& \left(\delta_{12}^{d}\right)_{L L} \sim \lambda^{4}, \quad\left(\delta_{13}^{d}\right)_{L L} \sim \lambda^{2}, \quad\left(\delta_{23}^{d}\right)_{L L} \sim \lambda^{2}, \\
& \left(\delta_{12}^{d}\right)_{R R} \sim \lambda^{4}, \quad\left(\delta_{13}^{d}\right)_{R R} \lesssim \lambda^{4}, \quad\left(\delta_{23}^{d}\right)_{R R} \lesssim \lambda^{4}, \\
& \left(\delta_{12}^{u}\right)_{L L} \sim \lambda^{4}, \quad\left(\delta_{13}^{u}\right)_{L L} \sim \lambda^{2}, \quad\left(\delta_{23}^{u}\right)_{L L} \sim \lambda^{2} \text {, } \\
& \left(\delta_{12}^{u}\right)_{R R} \sim \lambda^{4}, \quad\left(\delta_{13}^{u}\right)_{R R} \lesssim \lambda^{4}, \quad\left(\delta_{23}^{u}\right)_{R R} \lesssim \lambda^{4},
\end{aligned}
$$

at the Planck scale. In addition, we have flavor-blind renormalization group (RG) effects due to gaugino masses of $O\left(7 M_{1 / 2}^{2}\right)$. Such RG effects reduce the above mass insertion parameters by $O\left(10^{-1}\right)$, because gaugino masses $M_{1 / 2}$ are naturally of $O\left(m_{3 / 2}\right)$ within the framework of dilaton/moduli mediation. These values of mass insertion parameters satisfy experimental constraints on FCNCs $[13,14]$. When we derived Eq. (4.11), we assumed that $m_{11}^{2} / m_{33}^{2} \sim O(1)$. If the ratio is larger than $O(1 / \lambda)$, then the mass insertion parameters get enhanced by $1 / \lambda$. Still they are phenomenologically acceptable.

Furthermore, we have assumed extra $U(1)$ symmetries to obtain realistic Yukawa matrices. Breaking of such extra symmetries, in general, induces $D$-term contributions to soft scalar masses which are proportional to the $U(1)$ charges of fields, as shown in Eq. (B2) of Appendix B. (See Ref. [28] for heterotic models.) However, as a consequence of the $D_{4}$ flavor structure, such $D$-term contributions are also degenerate between the first and second families, because they must have the same $U(1)$ charges. Thus, after including such $D$-term contributions, SUSY breaking scalar mass-squared matrices are still of the form of Eq. (4.1). ${ }^{4}$

\section{B. $A$-terms}

Now, let us study the SUSY breaking trilinear scalar couplings, i.e. the $A$-terms. Soft trilinear terms are obtained as [27]

$$
\begin{aligned}
h_{i j k}= & \sum_{m} F^{m}\left[\partial_{m} Y_{i j k}+\partial_{m} \hat{K}-\sum_{n, p}\left(K^{\phi_{n} \phi_{p}^{\dagger}} \partial_{m} K_{\phi_{i} \phi_{p}^{\dagger}} Y_{n j k}\right.\right. \\
& \left.\left.+K^{\phi_{n} \phi_{p}^{\dagger}} \partial_{m} K_{\phi_{j} \phi_{p}^{\dagger}} Y_{i n k}+K^{\phi_{n} \phi_{p}^{\dagger}} \partial_{m} K_{\phi_{k} \phi_{p}^{\dagger}} Y_{i j n}\right)\right],
\end{aligned}
$$

in generic case with nonvanishing off-diagonal elements of the Kähler metric. When the Kähler metric of matter fields (within the framework of supergravity) is diagonal, the SUSY breaking trilinear scalar couplings are written as

$$
h_{i j k}=Y_{i j k} A_{i j k} \text {, }
$$

\footnotetext{
${ }^{4}$ Moreover, RG effects due to extra $U(1)$ gaugino masses are also significant [29]. Such RG effects are also degenerate between the first and second families in our model, because they have the same $U(1)$ charges.
}

$$
A_{i j k}=A_{i j k}^{(K)}+A_{i j k}^{(Y)}
$$

where

$$
\begin{gathered}
A_{i j k}^{(K)}=\sum_{m} F^{m}\left[\partial_{m} \hat{K}-\partial_{m} \ln \left(K_{\phi_{i} \phi_{i}^{\dagger}} K_{\phi_{j} \phi_{j}^{\dagger}} K_{\phi_{k} \phi_{k}^{\dagger}}\right)\right], \\
A_{i j k}^{(Y)}=\sum_{m} F^{m} \partial_{m} \ln \left(Y_{i j k}\right) .
\end{gathered}
$$

The first term of $A_{i j k}^{(K)}$ is the universal contribution due to the Kähler potential $\hat{K}$ of the dilaton and moduli fields. The second term of $A_{i j k}^{(K)}$ is the contribution through the wave function, i.e. the Kähler metric. The term $A_{i j k}^{(Y)}$ appears only when Yukawa couplings are field dependent. Prior to $D_{4}$ symmetry breaking, the only nonvanishing entry in the Yukawa matrix is for $Y_{i j k}$ with $i=j=3$ and $k=H$ (Higgs).

We consider the $A$-matrices after the $D_{4}$ symmetry breaking. As studied in Sec. IVA, the $D_{4}$ symmetry breaking induces off-diagonal elements of Kähler metric, e.g. for left-handed squarks,

$$
\left(\begin{array}{ccc}
O(1) & O\left(\lambda^{4}\right) & O\left(\lambda^{3}\right) \\
O\left(\lambda^{4}\right) & O(1) & O\left(\lambda^{2}\right) \\
O\left(\lambda^{3}\right) & O\left(\lambda^{2}\right) & O(1)
\end{array}\right)
$$

Right-handed squarks of up and down sectors have a similar form of Kähler metric, but $(i, 3)$ and $(3, i)$ elements for $i=1,2$ are more suppressed. The Kähler metric is almost diagonal, and such a diagonal form is violated by $O\left(\lambda^{4}\right)$ in the $(1,2)$ entry. Thus, it is reasonable to neglect off-diagonal elements of the Kähler metric in the first approximation. Then, as we discuss shortly, our model of Yukawa matrices discussed in the previous section leads to the following form of scalar trilinear coupling matrices,

$$
\begin{aligned}
h^{(u)} & =\left(\begin{array}{ccc}
O\left(\lambda^{4}\right) & O\left(\lambda^{4}\right) & O\left(\lambda^{3}\right) \\
O\left(\lambda^{4}\right) & O\left(\lambda^{4}\right) & O\left(\lambda^{2}\right) \\
0 & 0 & O(1)
\end{array}\right) \times A, \\
h^{(d)} & =\left(\begin{array}{ccc}
O\left(\lambda^{3}\right) & O\left(\lambda^{3}\right) & O\left(\lambda^{3}\right) \\
O\left(\lambda^{3}\right) & O\left(\lambda^{3}\right) & O\left(\lambda^{2}\right) \\
0 & 0 & O(1)
\end{array}\right) \times \lambda \times A .
\end{aligned}
$$

This seems to lead to mass insertion parameters, e.g.

$$
\left(\delta_{12}^{d}\right)_{L R} \sim \lambda^{3} \times m_{b} A / \tilde{m}^{2} .
$$

However, due to quark-squark mass alignment, we will show that our model actually leads to much smaller values of mass insertion parameters.

In the following we derive the scalar trilinear couplings in Eq. (4.18). For the moment, let us neglect off-diagonal elements of the Kähler metric as discussed above. Since the $D_{4}$ flavor structure requires that $K_{\phi_{1} \phi_{1}^{\dagger}}=K_{\phi_{2} \phi_{2}^{\dagger}}$, the form of $A^{(K)}$ including the Higgs field is obtained as 


$$
A_{i j H}^{(K)}=\left(\begin{array}{lll}
A_{0}^{(K)} & A_{0}^{(K)} & A_{1}^{(K)} \\
A_{0}^{(K)} & A_{0}^{(K)} & A_{1}^{(K)} \\
A_{1}^{\prime(K)} & A_{1}^{\prime(K)} & A_{3}^{(K)}
\end{array}\right),
$$

for both the up and down sectors.

Now, let us discuss the $A^{(Y)}$ part. The Yukawa couplings depend on gauge singlets $\sigma$ (3.3). Thus, their $F$-components $F^{\sigma}$ contribute to $A^{(Y)}$, and it is important to evaluate $F^{\sigma}$. Within the framework of supergravity, the $F$-component of $\sigma$ is written as

$$
F_{\sigma[R]}^{\dagger}=-e^{\langle K\rangle / 2}\left\langle K_{\sigma[R] \sigma[R]} \sigma_{[R]}^{\dagger} \hat{W}+\partial_{\sigma[R]} \hat{W}\right\rangle,
$$

where $K_{\sigma[R] \sigma[R]}$ denotes the Kähler metric of $\sigma_{[R]}$, and $\hat{W}$ is the nontrivial superpotential leading to SUSY breaking. The $D_{4}$ symmetry requires

$$
K_{\sigma\left[D_{1}\right] \sigma\left[D_{1}\right]}=K_{\sigma\left[D_{2}\right] \sigma\left[D_{2}\right]}
$$

as well as $K_{\sigma\left[D_{1}\right] \sigma\left[D_{2}\right]}=K_{\sigma\left[D_{2}\right] \sigma\left[D_{1}\right]}=0$. Here we assume that the nontrivial superpotential does not include $\sigma$. In this case, we can write

$$
\frac{F^{\sigma[R]}}{\left\langle\sigma_{[R]}\right\rangle}=-e^{\langle K\rangle / 2}\left\langle\hat{W}^{*}\right\rangle .
$$

Therefore, the total $A$-matrices of the up and down sectors have the form

$$
A_{i j H}^{(u, d)}=\left(\begin{array}{lll}
A_{0}^{(u, d)} & A_{0}^{(u, d)} & A_{1}^{(u, d)} \\
A_{0}^{(u, d)} & A_{0}^{(u, d)} & A_{1}^{(u, d)} \\
A_{1}^{\prime(u, d)} & A_{1}^{\prime(u, d)} & A_{3}^{(u, d)}
\end{array}\right) .
$$

The $(2 \times 2)$ submatrices for the first and second families are degenerate. That implies that when we write Yukawa matrices of our model as

$$
\begin{aligned}
y^{(u)} & =\left(\begin{array}{ccc}
c_{11}^{(u)} \lambda^{4} & c_{12}^{(u)} \lambda^{4} & c_{13}^{(u)} \lambda^{3} \\
c_{21}^{(u)} \lambda^{4} & c_{22}^{(u)} \lambda^{4} & c_{23}^{(u)} \lambda^{2} \\
0 & 0 & 1
\end{array}\right), \\
y^{(d)} & =\left(\begin{array}{ccc}
c_{11}^{(d)} \lambda^{3} & c_{12}^{(d)} \lambda^{3} & c_{13}^{(d)} \lambda^{3} \\
c_{21}^{(d)} \lambda^{3} & c_{22}^{(d)} \lambda^{3} & c_{23}^{(d)} \lambda^{2} \\
0 & 0 & 1
\end{array}\right) \times \lambda,
\end{aligned}
$$

in the $D_{4}$ basis, the scalar trilinear coupling matrices have the following form,

$$
\begin{aligned}
h^{(u)} & =\left(\begin{array}{ccc}
c_{11}^{(u)} \lambda^{4} & c_{12}^{(u)} \lambda^{4} & b^{(u)} c_{13}^{(u)} \lambda^{3} \\
c_{21}^{(u)} \lambda^{4} & c_{22}^{(u)} \lambda^{4} & b^{(u)} c_{23}^{(u)} \lambda^{2} \\
0 & 0 & c^{(u)}
\end{array}\right) \times A, \\
h^{(d)} & =\left(\begin{array}{ccc}
c_{11}^{(d)} \lambda^{3} & c_{12}^{(d)} \lambda^{3} & b^{(d)} c_{13}^{(d)} \lambda^{3} \\
c_{21}^{(d)} \lambda^{3} & c_{22}^{(d)} \lambda^{3} & b^{(d)} c_{23}^{(d)} \lambda^{2} \\
0 & 0 & c^{(d)}
\end{array}\right) \times \lambda \times A .
\end{aligned}
$$

as given in Eq. (4.18). Note, this form is quite different from one which is obtained in the $U(1)$ Froggatt-Nielsen model as shown in Appendix B.

Now consider the consequence of quark-squark mass alignment. The upper left $2 \times 2$ submatrices of $y^{(u)}\left(y^{(d)}\right)$ and $h^{(u)} \quad\left(h^{(d)}\right)$ are proportional to each other. We can multiply each of $y^{(u)}$ and $y^{(d)}$ by two unitary matrices on the left and the right-hand sides to diagonalize the upper left $2 \times 2$ submatrix. After doing this, we obtain

$$
\begin{aligned}
y^{(u)} & =\left(\begin{array}{ccc}
c_{11}^{\prime(u)} \lambda^{7} & 0 & c_{13}^{\prime(u)} \lambda^{2} \\
0 & c_{22}^{\prime(u)} \lambda^{4} & c_{23}^{\prime(u)} \lambda^{2} \\
0 & 0 & 1
\end{array}\right), \\
y^{(d)} & =\left(\begin{array}{ccc}
c_{11}^{\prime(d)} \lambda^{4} & 0 & c_{13}^{\prime(d)} \lambda^{2} \\
0 & c_{22}^{\prime(d)} \lambda^{3} & c_{23}^{\prime(d)} \lambda^{2} \\
0 & 0 & 1
\end{array}\right) \times \lambda .
\end{aligned}
$$

In the same basis, the $h$ matrices look like

$$
\begin{aligned}
h^{(u)} & =\left(\begin{array}{ccc}
c_{11}^{\prime(u)} \lambda^{7} & 0 & b^{(u)} c_{13}^{\prime(u)} \lambda^{2} \\
0 & c_{22}^{\prime(u)} \lambda^{4} & b^{(u)} c_{23}^{\prime(u)} \lambda^{2} \\
0 & 0 & c^{(u)}
\end{array}\right) \times A, \\
h^{(d)} & =\left(\begin{array}{ccc}
c_{11}^{\prime(d)} \lambda^{4} & 0 & b^{(d)} c_{13}^{(d)} \lambda^{2} \\
0 & c_{22}^{\prime(d)} \lambda^{3} & b^{(d)} c_{23}^{\prime(d)} \lambda^{2} \\
0 & 0 & c^{(d)}
\end{array}\right) \times \lambda \times A .
\end{aligned}
$$

Then, we can estimate the $L R$ and $R L$ mass insertion parameters applying the perturbative diagonalization formula to the above matrices,

$$
\begin{array}{rlrl}
\left(\delta_{12}^{d}\right)_{L R} & \sim \lambda^{7} \times m_{b} A / \tilde{m}^{2}, & & \left(\delta_{13}^{d}\right)_{L R} \sim \lambda^{2} \times m_{b} A / \tilde{m}^{2}, \\
\left(\delta_{23}^{d}\right)_{L R} \sim \lambda^{2} \times m_{b} A / \tilde{m}^{2}, & & \left(\delta_{12}^{d}\right)_{R L} \sim \lambda^{8} \times m_{b} A / \tilde{m}^{2}, \\
\left(\delta_{13}^{d}\right)_{R L} \sim \lambda^{6} \times m_{b} A / \tilde{m}^{2}, & & \left(\delta_{23}^{d}\right)_{R L} \sim \lambda^{5} \times m_{b} A / \tilde{m}^{2}, \\
\left(\delta_{12}^{u}\right)_{L R} \sim \lambda^{8} \times m_{t} A / \tilde{m}^{2}, & & \left(\delta_{13}^{u}\right)_{L R} \sim \lambda^{2} \times m_{t} A / \tilde{m}^{2}, \\
\left(\delta_{23}^{u}\right)_{L R} \sim \lambda^{2} \times m_{t} A / \tilde{m}^{2}, & & \left(\delta_{12}^{u}\right)_{R L} \sim \lambda^{11} \times m_{t} A / \tilde{m}^{2}, \\
\left(\delta_{13}^{u}\right)_{R L} \sim \lambda^{9} \times m_{t} A / \tilde{m}^{2}, & & \left(\delta_{23}^{u}\right)_{R L} \sim \lambda^{6} \times m_{t} A / \tilde{m}^{2} .
\end{array}
$$

As noted previously, these are much smaller than their naive values, Eq. (4.19). They satisfy experimental constraints [13,14]. When we derived Eq. (4.29), we assumed that all the $A$ 's in Eq. (4.24) have almost the same sizes, and all of their ratios are of $O(1)$. If their ratios are larger than $O(1 / \lambda)$, then the above estimate should be multiplied by $O(1 / \lambda)$, which is still phenomenologically viable.

Up until now we have neglected off-diagonal elements of the Kähler metric. Here we discuss corrections due to these neglected terms. Such corrections violate the degeneracy of the upper left $(2 \times 2)$ submatrices in $A_{i j H}^{(u, d)}(4.24)$ by $O\left(\lambda^{4}\right)$. Similarly, they can make corrections to other entries. Note that Eqs. (4.13), (4.14), (4.15), and (4.16) are not available for nonvanishing off-diagonal elements of the Kähler metric and we have to use the generic formula 
(4.12). Including these corrections modifies the scalar trilinear couplings to

$$
\begin{aligned}
h^{(u)} & =\left(\begin{array}{ccc}
{\left[c_{11}^{(u)}+O\left(\lambda^{4}\right)\right] \lambda^{4}} & {\left[c_{12}^{(u)}+O\left(\lambda^{4}\right)\right] \lambda^{4}} & {\left[b^{(u)} c_{13}^{(u)}+O\left(\lambda^{3}\right)\right] \lambda^{3}} \\
{\left[c_{21}^{(u)}+O\left(\lambda^{4}\right)\right] \lambda^{4}} & {\left[c_{22}^{(u)}+O\left(\lambda^{4}\right)\right] \lambda^{4}} & {\left[b^{(u)} c_{23}^{(u)}+O\left(\lambda^{4}\right)\right] \lambda^{2}} \\
O\left(\lambda^{6}\right) & O\left(\lambda^{6}\right) & c^{(u)}
\end{array}\right) \times A, \\
h^{(d)} & =\left(\begin{array}{ccc}
{\left[c_{11}^{(d)}+O\left(\lambda^{4}\right)\right] \lambda^{3}} & {\left[c_{12}^{(d)}+O\left(\lambda^{4}\right)\right] \lambda^{3}} & {\left[b^{(d)} c_{13}^{(d)}+O\left(\lambda^{3}\right)\right] \lambda^{3}} \\
{\left[c_{21}^{(d)}+O\left(\lambda^{4}\right)\right] \lambda^{3}} & {\left[c_{22}^{(d)}+O\left(\lambda^{4}\right)\right] \lambda^{3}} & {\left[b^{(d)} c_{23}^{(d)}+O\left(\lambda^{4}\right)\right] \lambda^{2}} \\
O\left(\lambda^{6}\right) & O\left(\lambda^{6}\right) & c^{(d)}
\end{array}\right) \times \lambda \times A .
\end{aligned}
$$

Corrections such as these do not drastically change the above estimation of mass insertion parameters.

\section{CONCLUSIONS}

In this paper we have discussed the structure of heterotic string models with the discrete non-Abelian flavor symmetry $D_{4}$. Such flavor symmetries are easily obtained in a variety of orbifold constructions of the heterotic string [20-23]. For example, $D_{4}$ flavor symmetries are easily obtained in $Z_{2} \times Z_{N}$ orbifold constructions. We have also shown that these discrete non-Abelian flavor symmetries may be useful for understanding the hierarchy of fermion masses and mixing angles. In addition, they constrain the SUSY breaking mass-squared matrices and cubic scalar interaction matrices; hence suppressing flavor violating processes. In particular, the non-Abelian flavor symmetries, with quarks of the first two families in one irreducible representation, reduce the sensitivity to flavor violating interactions. In Appendix B, we have also compared the structure of soft SUSY breaking mass-squared matrices and $A$-term matrices consistent with discrete non-Abelian flavor symmetries and those of $U(1)$ flavor symmetries. As discussed, $U(1)$ flavor symmetries do not sufficiently constrain FCNC processes.

We have considered only the quark sector. We can extend the previous analysis to the lepton sector. Indeed, we can obtain the same results for Yukawa matrices, SUSY breaking scalar masses, and $A$-terms as Eqs. (3.3), (4.1), and (4.24). With a $D_{4}$ flavor symmetry, there is no difficulty encountered for obtaining the standard seesaw mechanism with heavy right-handed neutrinos. However there are still many more additional parameters due to the right-handed Majorana masses. The number of free parameters is typically larger than the number of observables. Thus, we have no prediction in the Yukawa sector, unless we impose texture zeros or assume additional symmetries. On the other hand, we have predictions for SUSY breaking terms. Actually, the branching ratio for $\mu \rightarrow e \gamma$ leads to the strongest constraint. Hence, the degeneracy between the first and second families would help to satisfy this constraint, in particular, by suppressing the factor, $\left(\delta_{12}^{\ell}\right)_{L L}$.

In order to obtain more predictive Yukawa sectors we need to reduce the number of free parameters. This can be done by embedding the flavor structure into GUTs. In some orbifold GUT and string models with an intermediate Pati-
Salam gauge symmetry, the third generation Yukawa couplings are unified with $\lambda_{t}=\lambda_{b}=\lambda_{\tau}=\lambda_{\nu_{\tau}}$. However in order for these theories to be phenomenologically acceptable, certain relations among the soft SUSY breaking terms must hold, in particular $A_{33} \approx-2 m_{33}$ [30]. It is clear that this relation is not satisfied with simple scenarios of dilaton or moduli SUSY breaking. A possible explanation may require a more complicated SUSY breaking scenario, for example, see Appendix C.

\section{ACKNOWLEDGMENTS}

We would like to acknowledge R.-J. Zhang who participated during the early stages of this work. T.K. is supported in part by the Grand-in-Aid for Scientific Research No. 17540251 and the Grant-in-Aid for the 21st Century COE "The Center for Diversity and Universality in Physics" from the Ministry of Education, Culture, Sports, Science and Technology of Japan. S. R. acknowledges partial support under DOE Contract No. DOE/ER/ 01545-865. J.-h. P. was supported by JSPS and Grant-inAid No. 17.05302. P. K. was supported by KOSEF SRC program through CHEP at Kyungpook National University.

\section{APPENDIX A: $D_{4}$ DISCRETE GROUP}

The $D_{4}$ discrete group has five representations including a doublet $D$, a trivial singlet $A_{1}$, and three nontrivial singlets $B_{1}, B_{2}, A_{2}$, which are shown in Table III.

A product of two doublets is decomposed as four singlets,

$$
(D \times D)=A_{1}+B_{1}+B_{2}+A_{2} .
$$

More explicitly, we consider two $D_{4}$ doublets $S_{A}$ and $\bar{S}_{A}$ $(A=1,2)$. Their product $S_{A} \bar{S}_{B}$ is decomposed in terms of

TABLE III. Representations of $D_{4}$ symmetry.

\begin{tabular}{lccccc}
\hline \hline Representations & $I$ & $-I$ & $\pm \sigma_{1}$ & $\pm \sigma_{3}$ & $\mp i \sigma_{2}$ \\
\hline Doublet- $D$ & 2 & -2 & 0 & 0 & 0 \\
Singlet- $A_{1}$ & 1 & 1 & 1 & 1 & 1 \\
Singlet $-B_{1}$ & 1 & 1 & 1 & -1 & -1 \\
Singlet- $B_{2}$ & 1 & 1 & -1 & 1 & -1 \\
Singlet- $A_{2}$ & 1 & 1 & -1 & -1 & 1 \\
\hline \hline
\end{tabular}


$A_{1}, B_{1}, B_{2}, A_{2}$,

$$
\begin{aligned}
& S_{1} \bar{S}_{1}+S_{2} \bar{S}_{2} \sim A_{1}, \\
& S_{1} \bar{S}_{2}+S_{2} \bar{S}_{1} \sim B_{1}, \\
& S_{1} \bar{S}_{1}-S_{2} \bar{S}_{2} \sim B_{2}, \\
& S_{1} \bar{S}_{2}-S_{2} \bar{S}_{1} \sim A_{2} .
\end{aligned}
$$

\section{APPENDIX B: SUSY BREAKING TERMS IN THE $U(1)$ FROGGATT-NIELSEN MODEL}

One of the famous flavor mechanisms is the $U(1)$ Froggatt-Nielsen mechanism in the string-inspired approach. Here we give brief comments on soft SUSY breaking terms to compare them with our results from the $D_{4}$ flavor structure.

In the simple $U(1) \mathrm{FN}$ mechanism, the effective Yukawa couplings are obtained through the higher dimensional operators, e.g.

$$
\chi^{q_{Q i}+q_{u j}+q_{H u}} Q_{i} u_{j} H_{u},
$$

where $\chi$ is the FN field with nonvanishing VEV and $q_{Q i}$, $q_{u j}$, and $q_{H u}$ are extra $U(1)$ charges of $Q_{i}, u_{j}$, and $H_{u}$, respectively. In this mechanism, it is easy to derive realistic Yukawa matrices.

We have the usual sfermion masses due to $F$-components of moduli fields like Eqs. (4.4). Since the $U(1)$ flavor symmetry, in general, does not specify the Kähler metric of matter fields, one can not give a generic statement on this part. In addition to this usual part, the extra $U(1)$ breaking induces additional contributions to sfermion masses, that is, the so-called $D$-term contributions. Such part is, in general, written as

$$
\Delta m_{\alpha}^{2}=q_{\alpha} m_{D}^{2},
$$

where $q_{\alpha}$ is the extra $U(1)$ charge of matter field, and $m_{D}^{2}$ itself is universal for all matter fields, that is, these are proportional to extra $U(1)$ charges. (See Ref. [28] for heterotic models.) Furthermore, RG effects due to extra $U(1)$ gaugino masses may generate significant nondegeneracy when each family has different $U(1)$ charges [29].

As Sec. IV B, A-terms are obtained by calculating Eqs. (4.15) and (4.16). In this case, we obtain the $A^{(Y)}$-matrices [31,32],

$$
A_{i j H}=\frac{F^{\chi}}{\chi}\left(q_{Q i}+q_{u j}+q_{H}\right),
$$

for $Y_{i j H}=\chi^{q_{Q_{i}}+q_{u j}+q_{H}}$. Here note that $\frac{F \chi}{\chi}=O\left(m_{3 / 2}\right)$ because $F^{\chi}=\chi \bar{W}$. Also note that the flavor dependence in the second term of $A^{(K)}$ can be separated. As a result, $A$-terms are decomposed as [33]

$$
A_{\alpha \beta H}=A_{\alpha}^{L}+A_{\beta}^{R} \text {. }
$$

Moreover, the trilinear scalar coupling matrix $h_{i j H}$ is written as

$$
\begin{aligned}
h_{i j H}= & Y_{i j} A_{i j H} \\
= & \left(Y_{i j}\right) \cdot\left(\begin{array}{lll}
A_{1}^{R} & & \\
& A_{2}^{R} & \\
& & A_{3}^{R}
\end{array}\right) \\
& +\left(\begin{array}{lll}
A_{1}^{L} & & \\
& A_{2}^{L} & \\
& & A_{3}^{L}
\end{array}\right) \cdot\left(Y_{i j}\right) .
\end{aligned}
$$

This form of the $A$-matrices, in general, leads to dangerous FCNC effects.

\section{APPENDIX C: $S O(10)$ YUKAWA UNIFICATION AND SUSY BREAKING TERMS}

$S O(10)$ Yukawa unification for the third family, resulting from the renormalizable coupling

$$
W \supset \lambda 16_{3} 1016_{3} \text {, }
$$

gives

$$
\lambda_{t}=\lambda_{b}=\lambda_{\tau}=\lambda_{\nu_{\tau}}=\lambda .
$$

In order to fit the top, bottom, and tau masses at the weak scale, it has been shown that it is necessary to be in a particular region of soft SUSY breaking parameter space [30]. Define the soft SUSY breaking parameters: $A_{0}$, the cubic scalar interaction mass term; $M_{1 / 2}$, a universal gaugino mass; $m_{16}$, the soft scalar mass for squarks and sleptons; and $m_{10}$, the Higgs soft SUSY breaking mass. Then we require the relation

$$
\begin{aligned}
& A_{0}=-2 m_{16}, \\
& m_{10}=\sqrt{2} m_{16},
\end{aligned}
$$

and

$$
\mu \sim M_{1 / 2} \ll m_{16} .
$$

The question is can this relation come naturally in string theory. It is difficult to obtain this result from a combination of dilaton and T moduli SUSY breaking. Here we argue that this simple relation can come from $D$-term and minimal supersymmetric standard model (MSSM) singlet SUSY breaking. Consider the $U(1)_{X}$ symmetry in $E_{6}$ which commutes with $S O(10)$. The 27 dimensional representation of $E_{6}$ decomposes under $S O(10) \times U(1)_{X}$ as

$$
27 \rightarrow(16,1) \oplus(10,-2) \oplus(1,4) .
$$

If we now assume the $\mathbf{1 6}$ of quarks and leptons comes from a $\mathbf{2 7}$, while the Higgs doublets come from a $\overline{\mathbf{2 7}}$, we obtain the wanted relation

$$
m_{10}^{2}=2 m_{16}^{2} \equiv 2 D_{X} .
$$

We also obtain the same scalar mass for all three families of squarks and sleptons, consistent with minimal flavor 
violation. Furthermore, the relation

$$
\mu \sim M_{1 / 2} \ll m_{16}
$$

is easy to accommodate, for example, by subdominant dilaton SUSY breaking. Thus the only remaining question is the origin of the cubic scalar parameter $A_{0}$.

Assume we have a term in the superpotential of the form

$W \supset \chi^{Q_{X}\left(Q_{i}\right)+Q_{X}\left(u_{j}\right)+Q_{X}\left(H_{u}\right)} Q_{i} u_{j} H_{u}=\chi^{4} Q_{i} u_{j} H_{u}$, where $\chi$ (with $Q_{X}(\chi)=-1$ ) is a MSSM singlet field. Then in order to obtain the relation (Eq. (C3)) from Eq. (B3) we need

$$
A_{0}=4 F_{\chi} / \chi \approx-2 \sqrt{D_{X}}
$$

This relation may also be accommodated.
[1] K. S. Babu and S. M. Barr, Phys. Lett. B 381, 202 (1996); C. H. Albright and S. M. Barr, Phys. Rev. D 58, 013002 (1998); C. H. Albright, K. S. Babu, and S. M. Barr, Phys. Rev. Lett. 81, 1167 (1998); J. Sato and T. Yanagida, Phys. Lett. B 430, 127 (1998); N. Irges, S. Lavignac, and P. Ramond, Phys. Rev. D 58, 035003 (1998); C. H. Albright and S. M. Barr, Phys. Lett. B 452, 287 (1999); Y. Nomura and T. Yanagida, Phys. Rev. D 59, 017303 (1998); N. Haba, Phys. Rev. D 59, 035011 (1999); G. Altarelli and F. Feruglio, J. High Energy Phys. 11 (1998) 021; Z. Berezhiani and A. Rossi, J. High Energy Phys. 03 (1999) 002; K. Hagiwara and N. Okamura, Nucl. Phys. B548, 60 (1999); G. Altarelli and F. Feruglio, Phys. Lett. B 451, 388 (1999); K. S. Babu, J. Pati, and F. Wilczek, Nucl. Phys. B566, 33 (2000); M. Bando and T. Kugo, Prog. Theor. Phys. 101, 1313 (1999); K. Izawa, K. Kurosawa, N. Nomura, and T. Yanagida, Phys. Rev. D 60, 115016 (1999); P. Frampton and A. Rasin, Phys. Lett. B 478, 424 (2000); R. Barbieri, G. Kane, L. J. Hall, and G. G. Ross, arXiv:hep-ph/9901228; M. Bando, T. Kugo, and K. Yoshioka, Prog. Theor. Phys. 104, 211 (2000); R. Kitano and Y. Mimura, Phys. Rev. D 63, 016008 (2000).

[2] R. Dermisek, Phys. Rev. D 70, 073016 (2004).

[3] R. Dermisek and S. Raby, Phys. Lett. B 622, 327 (2005).

[4] P. H. Frampton and T. W. Kephart, Int. J. Mod. Phys. A 10, 4689 (1995).

[5] A. Pomarol and D. Tommasini, Nucl. Phys. B466, 3 (1996); R. Barbieri, G. R. Dvali, and L. J. Hall, Phys. Lett. B 377, 76 (1996); R. Barbieri, L. J. Hall, S. Raby, and A. Romanino, Nucl. Phys. B493, 3 (1997); T. Blazek, S. Raby, and K. Tobe, Phys. Rev. D 62, 055001 (2000).

[6] S. Pakvasa and H. Sugawara, Phys. Lett. B 73, 61 (1978); L. J. Hall and H. Murayama, Phys. Rev. Lett. 75, 3985 (1995); C. D. Carone, L. J. Hall, and H. Murayama, Phys. Rev. D 53, 6282 (1996); C.D. Carone and R. F. Lebed, Phys. Rev. D 60, 096002 (1999); R. Dermisek and S. Raby, Phys. Rev. D 62, 015007 (2000); J. Kubo, A. Mondragon, M. Mondragon, and E. Rodriguez-Jauregui, Prog. Theor. Phys. 109, 795 (2003); J. Kubo, Phys. Lett. B 578, 156 (2004); S. L. Chen, M. Frigerio, and E. Ma, Phys. Rev. D 70, 073008 (2004); K. Hamaguchi, M. Kakizaki, and M. Yamaguchi, Phys. Rev. D 68, 056007 (2003); T. Kobayashi, J. Kubo, and H. Terao, Phys. Lett. B 568, 83 (2003); N. Haba and K. Yoshioka, Nucl. Phys. B739, 254
(2006); M. Honda and M. Tanimoto, Phys. Rev. D 75, 096005 (2007).

[7] D. Wyler, Phys. Rev. D 19, 3369 (1979); E. Ma and G. Rajasekaran, Phys. Rev. D 64, 113012 (2001); E. Ma, Mod. Phys. Lett. A 17, 627 ( 2002); K. S. Babu, E. Ma, and J. W. F. Valle, Phys. Lett. B 552, 207 (2003); E. Ma, Phys. Rev. D 70, 031901 (2004); K. S. Babu, T. Kobayashi, and J. Kubo, Phys. Rev. D 67, 075018 (2003); G. Altarelli and F. Feruglio, Nucl. Phys. B720, 64 (2005); A. Zee, Phys. Lett. B 630, 58 (2005); G. Altarelli and F. Feruglio, Nucl. Phys. B741, 215 (2006).

[8] W. Grimus, A. S. Joshipura, S. Kaneko, L. Lavoura, and M. Tanimoto, J. High Energy Phys. 07 (2004) 078.

[9] K. S. Babu and J. Kubo, Phys. Rev. D 71, 056006 (2005).

[10] C. Hagedorn, M. Lindner, and F. Plentinger, Phys. Rev. D 74, 025007 (2006).

[11] D. B. Kaplan and M. Schmaltz, Phys. Rev. D 49, 3741 (1994); K. C. Chou and Y. L. Wu, arXiv:hep-ph/9708201; I. de Medeiros Varzielas, S. F. King, and G. G. Ross, Phys. Lett. B 644, 153 (2007).

[12] M. Dine, R. G. Leigh, and A. Kagan, Phys. Rev. D 48, 4269 (1993); Y. Nir and N. Seiberg, Phys. Lett. B 309, 337 (1993); M. Leurer, Y. Nir, and N. Seiberg, Nucl. Phys. B398, 319 (1993); P. Pouliot and N. Seiberg, Phys. Lett. B 318, 169 (1993); D. B. Kaplan and M. Schmaltz, Phys. Rev. D 49, 3741 (1994); A. Pomarol and D. Tommasini, Nucl. Phys. B466, 3 (1996); R. Barbieri, G. R. Dvali, and L. J. Hall, Phys. Lett. B 377, 76 (1996).

[13] F. Gabbiani, E. Gabrielli, A. Masiero, and L. Silvestrini, Nucl. Phys. B477, 321 (1996).

[14] P. Ko, J. h. Park, and G. Kramer, Eur. Phys. J. C 25, 615 (2002).

[15] P. H. Chankowski, O. Lebedev, and S. Pokorski, Nucl. Phys. B717, 190 (2005).

[16] L. E. Ibanez, J. E. Kim, H. P. Nilles, and F. Quevedo, Phys. Lett. B 191, 282 (1987).

[17] A. Font, L. E. Ibanez, H. P. Nilles, and F. Quevedo, Phys. Lett. B 210, 101 (1988); 213, 274 (1988).

[18] J. A. Casas and C. Munoz, Phys. Lett. B 214, 63 (1988).

[19] J. A. Casas and C. Munoz, Phys. Lett. B 212, 343 (1988).

[20] T. Kobayashi, S. Raby, and R. J. Zhang, Phys. Lett. B 593, 262 (2004).

[21] T. Kobayashi, S. Raby, and R. J. Zhang, Nucl. Phys. B704, 3 (2005). 
[22] S. Förste, H.P. Nilles, P. K. S. Vaudrevange, and A. Wingerter, Phys. Rev. D 70, 106008 (2004);S. Förste, H. P. Nilles, and A. Wingerter, Phys. Rev. D 72, 026001 (2005); 73, 066011 (2006); W. Buchmüller, K. Hamaguchi, O. Lebedev, and M. Ratz, Nucl. Phys. B712, 139 (2005); Phys. Rev. Lett. 96, 121602 (2006); arXiv:hep-th/0606187; K. S. Choi, S. Groot Nibbelink, and M. Trapletti, J. High Energy Phys. 12 (2004) 063; H. P. Nilles, S. Ramos-Sanchez, P. K. S. Vaudrevange, and A. Wingerter, J. High Energy Phys. 04 (2006) 050; O. Lebedev, H. P. Nilles, S. Raby, S. Ramos-Sanchez, M. Ratz, P. K. S. Vaudrevange, and A. Wingerter, Phys. Lett. B 645, 88 (2007); arXiv:hep-th/0611203; J. E. Kim and B. Kyae, arXiv:hep-th/0608085; arXiv:hep-th/0608086.

[23] T. Kobayashi, H. P. Nilles, F. Ploger, S. Raby, and M. Ratz, Nucl. Phys. B768, 135 (2007).

[24] A. Brignole, L.E. Ibáñez, and C. Muñoz, Nucl. Phys. B422, 125 (1994); B436, 747(E) (1995).

[25] T. Kobayashi, D. Suematsu, K. Yamada, and Y.
Yamagishi, Phys. Lett. B 348, 402 (1995).

[26] A. Brignole, L. E. Ibáñez, C. Muñoz, and C. Scheich, Z. Phys. C 74, 157 (1997).

[27] V. S. Kaplunovsky and J. Louis, Phys. Lett. B 306, 269 (1993).

[28] Y. Kawamura and T. Kobayashi, Phys. Lett. B 375, 141 (1996); 388, 867(E) (1996); Phys. Rev. D 56, 3844 (1997).

[29] T. Kobayashi, H. Nakano, H. Terao, and K. Yoshioka, Prog. Theor. Phys. 110, 247 (2003); K. S. Babu, T. Enkhbat, and I. Gogoladze, Nucl. Phys. B678, 233 (2004).

[30] T. Blazek, R. Dermisek, and S. Raby, Phys. Rev. Lett. 88, 111804 (2002); Phys. Rev. D 65, 115004 (2002); K. Tobe and J. D. Wells, Nucl. Phys. B663, 123 (2003); D. Auto, H. Baer, C. Balazs, A. Belyaev, J. Ferrandis, and X. Tata, J. High Energy Phys. 06 (2003) 023.

[31] S. Abel, S. Khalil, and O. Lebedev, Phys. Rev. Lett. 89, 121601 (2002).

[32] G. G. Ross and O. Vives, Phys. Rev. D 67, 095013 (2003).

[33] T. Kobayashi and O. Vives, Phys. Lett. B 506, 323 (2001). 